American Journal of Infectious Diseases 4 (1): 69-75, 2008

ISSN 1553-6203

(C) 2008 Science Publications

\title{
Patient and Mouse Antibodies against Dengue Virus Nonstructural Protein 1 Cross-React with Platelets and Cause Their Dysfunction or Depletion
}

\author{
${ }^{1,2}$ Chiou-Feng Lin, ${ }^{2}$ Huan-Yao Lei, ${ }^{3}$ Ching-Chuan Liu, ${ }^{2}$ Hsiao-Sheng Liu, ${ }^{4}$ Trai-Ming Yeh, \\ ${ }^{5}$ Robert Anderson and ${ }^{2}$ Yee-Shin Lin \\ ${ }^{1}$ Institute of Clinical Medicine, ${ }^{2}$ Department of Microbiology and Immunology, \\ ${ }^{3}$ Department of Pediatrics, ${ }^{4}$ Department of Medical Technology, \\ National Cheng Kung University Medical College, Tainan, Taiwan \\ ${ }^{5}$ Department of Microbiology and Immunology, Dalhousie University, \\ Halifax, Nova Scotia, Canada
}

\begin{abstract}
Thrombocytopenia is a clinical manifestation in dengue virus (DV) infection, yet its pathogenic mechanisms are unresolved. We previously showed that dengue patient sera contained antibodies cross-reactive with platelets. In this study, we demonstrated that the anti-platelet activity of dengue patient sera was due to the antibodies against DV nonstructural protein 1 (NS1). Studies using DV-infected or recombinant NS1-immunized mouse sera showed that anti-NS1 antibodies cross-reacted with human platelets. The platelet-binding activity of dengue patient sera or anti-NS1 antibodies was inhibited by treatment of platelets with anti-NS1 or patient sera. Further investigation showed that anti-NS1 antibodies were able to inhibit platelet aggregation and cause platelet lysis in the presence of complement. The platelet-binding activity and the induction of platelet lysis mediated by dengue patient sera or anti-NS1 antibodies were increased when platelets were activated by ADP or thrombin. Taken together, anti-NS1 antibodies account for the cross-reactivity with platelets and cause platelet dysfunction or depletion, which may be involved in the pathogenesis of dengue diseases.
\end{abstract}

Key words: Autoantibody, dengue virus, platelet, NS1

\section{INTRODUCTION}

Dengue virus (DV), an important human pathogenic flavivirus, causes serious epidemic disease ${ }^{[1]}$. Infection with DV manifests a range of symptoms from mild, self-limiting, febrile illness as dengue fever to a life-threatening dengue hemorrhagic fever and dengue hemorrhagic shock syndrome (DHF/DSS) ${ }^{[2,3]}$. In addition to the direct cell damage caused by virus-specified factors, host immune responses to virus infection may also contribute to the severity of dengue disease. For example, the effect of antibody-dependent enhancement (ADE) of DV infection is widely believed to be involved in the disease pathogenesis ${ }^{[3-8]}$. Furthermore, immunological and/or immunopathological mechanisms, including cytokine and chemokine production, immune cell and complement activation and apoptotic cell death may also contribute to disease progression ${ }^{[9-18]}$. The onset of autoimmune responses to DV infection may also contribute to dengue disease pathogenesis ${ }^{[10,19-21]}$.

Thrombocytopenia is a clinical feature of hematological abnormality in dengue patients ${ }^{[22,23]}$. An impaired hematopoietic progenitor cell proliferation by DV may cause a decrease in platelet counts ${ }^{[24,25]}$. Moreover, an increased antibody-dependent DV binding to platelets ${ }^{[26]}$ and an increased platelet binding to endothelial cells ${ }^{[27]}$ may also play roles in the pathogenesis of thrombocytopenia. The presence of cross-reactive antibodies in dengue patient sera causing platelet lysis also provide a potential mechanism for platelet loss ${ }^{[28]}$.

The manifestation of thrombocytopenia and the generation of anti-platelet antibody in DV-infected mice provide a model for the pathogenicity of dengue disease $^{[29]}$. Mouse antibodies against DV nonstructural protein 1 (NS1) can cross-react with human thrombocytes ${ }^{[19]}$. Our previous studies ${ }^{[28]}$ demonstrated that antibodies reactive with platelets were present in dengue patient sera. Further investigation showed the

Corresponding Author: Dr. Yee-Shin Lin, Department of Microbiology and Immunology, National Cheng Kung University Medical College, Tainan, Taiwan 
capacity of anti-platelet autoantibodies to inhibit platelet aggregation and to cause platelet cytotoxicity in the presence of complement. In the present study, we demonstrate that anti-NS1 antibodies account for the cross-reactivity of patient sera with platelets. We show further that anti-NS1 antibodies generated in mice exhibit binding activity with human platelets and that anti-NS1 antibodies have effects on platelet aggregation and platelet lysis.

\section{MATERIALS AND METHODS}

Patient sera: Sera were collected from 9 dengue patients (7 males and 2 females, with ages ranging from 7 to 57 years) showing clinical symptoms of fever, rash and bone pain during an outbreak of DV3 infection in southern Taiwan from November 1998 to January 1999 as reported previously ${ }^{[28]}$. Among those patients in this outbreak, five patients with dengue fever and four with DHF, DSS, or both, whose sera were collected from acute stage (3-7 days after fever onset) were included in this study. Normal control sera from five healthy individuals were used as background.

Mice: BALB/cByJ breeder mice were purchased from The Jackson Laboratory and maintained on standard laboratory food and water in the Laboratory Animal Center of National Cheng Kung University Medical College. Their 8-week-old progeny were used for the experiments.

Virus preparation and infection: DV2 (strain PL046) was propagated in the mosquito $\mathrm{C} 6 / 36$ cell line and stored at $-80^{\circ} \mathrm{C}$ until use. The titer of virus stock was adjusted to $1 \times 10^{11} \mathrm{PFU} \mathrm{mL} \mathrm{m}^{-1}$. Mice were inoculated intravenously with $1 \times 10^{8} \mathrm{PFU} /$ mouse of DV2 and their sera were collected after 8 days.

Preparation of recombinant NS1 and generation of anti-NS1 antibodies: Recombinant NS1 proteins were prepared as previously described ${ }^{[28]}$. The DV2 NS1 cDNA was cloned to pRSET $\mathrm{B}$ expression vector (Invitrogen) to establish a pRSET-DVNS1 plasmid. After the plasmid was introduced into Escherichia coli BL21(DE3) pLys strain (Invitrogen), the recombinant NS1 proteins were then induced by $2 \mathrm{mM}$ final concentration of IPTG and purified with TALON metal affinity resin (Clontech). The $45-\mathrm{kDa}$ NS1 protein was detected by SDS-PAGE and the protein sequence was confirmed by an Applied Biosystems 477A autosequencer. BALB/c mice were immunized intraperitoneally with $25 \mu \mathrm{g}$ of purified recombinant NS1 proteins emulsified in complete Freund's adjuvant (Gibco BRL) and then four times in incomplete Freund's adjuvant. Sera were obtained four days after the last immunization. The IgG fractions from hyperimmunized mouse sera were purified with protein A-sepharose affinity chromatography column (Pharmacia) and recovered with $\mathrm{HCl}$-glycine. The reactivity of purified IgG against NS1 was confirmed by SDS-PAGE and Western blot. The control IgG was eluted from protein A column loaded with normal mouse sera.

Detection of anti-platelet antibodies: Human peripheral blood collected in $0.11 \mathrm{M}$ sodium citrate $(9: 1)$ was centrifuged at $100 \times g$ for $10 \mathrm{~min}$ at room temperature. The upper layer as platelet-rich plasma (PRP) was removed to a $15-\mathrm{mL}$ tube, mixed with $0.34 \%$ EDTA in phosphate-buffered saline (PBS) and centrifuged at $1000 \times g$ for $10 \mathrm{~min}$. The pellets were washed 3 times with $0.34 \%$ EDTA in PBS and fixed in $10 \mathrm{~mL}$ of $1 \%$ formaldehyde in PBS at room temperature for $10 \mathrm{~min}$. The fixed platelet suspension was centrifuged at $1000 \times g$ for $10 \mathrm{~min}$ at room temperature. The pellets were washed in PBS twice and resuspended in $2 \mathrm{~mL}$ of PBS. The platelet count was determined using a hemocytometer.

For the detection of anti-platelet antibodies, $2.5 \times 10^{6}$ platelets were incubated with 1:25 dilution of patient sera or various doses of antibodies for $60 \mathrm{~min}$ on ice, then washed twice with PBS. The secondary antibodies, i.e., $20 \mu \mathrm{L}$ of FITC-conjugated anti-human IgG or IgM (Pharmingen) or $1 \mu \mathrm{L}$ of FITC-conjugated anti-mouse IgG or IgM (Cappel), were added and the mixture was incubated for $40 \mathrm{~min}$ on ice. After washing twice with PBS, the platelets were resuspended in PBS and analyzed by flow cytometry (FACScan; BD Biosciences) with excitation set at $488 \mathrm{~nm}$.

Platelet aggregation assay: The PRP was prepared as described above. After 10 min centrifugation at $100 \times g$, the lower layer was subsequently centrifuged at $1000 \times g$ for $10 \mathrm{~min}$ and the supernatant was collected as the platelet-poor plasma (PPP). The PRP was diluted to $10^{7}$ platelets in $450 \mu \mathrm{L}$ of PPP and various doses of anti-NS1 or control $\mathrm{IgG}$ was added and incubated at $37^{\circ} \mathrm{C}$ for $30 \mathrm{~min}$.

Platelets were induced to aggregate by addition of adenosine 5'-diphosphate (ADP; $20 \mu \mathrm{M}$ final concentration) with stirring at $37^{\circ} \mathrm{C}$ and measured in an automated aggregometer (PACKS-4, Platelet Aggregation Chromogenic Kinetic System, Helena Laboratories). The normal control (PRP alone) without the addition of antibodies was normalized to $100 \%$ of 
platelet aggregation and the PPP was used as blank control.

Platelet lysis assay: Various doses of anti-NS1 or control IgG were incubated with Low-Tox-M rabbit complement (Cedarlane Laboratories) at $37^{\circ} \mathrm{C}$ for $60 \mathrm{~min}$ before addition to the PRP. The PRP was prepared as described above and $10^{5}$ platelets in PBS were added into each well of 96-well ELISA plate. After incubation for 4 $\mathrm{h}$, the level of lactate dehydrogenase (LDH) activity in the culture supernatant was determined at $490 \mathrm{~nm}$ using a Cytotoxicity Detection Kit (Boehringer Mannheim). The percentage of cytotoxicity $=$ (experimental value-low control $) \div$ (high control-low control $) \times 100$. The low control was the level of spontaneous LDH release of PBS-treated group and the high control was the maximum LDH release of $2 \%$ Triton X-100-treated group.

Statistics: We used the paired $t$-test for statistical analysis. Statistical significance was set at $\mathrm{p}<0.05$.

\section{RESULTS}

Platelet-binding activity of dengue patient sera can be inhibited by preabsorption with NS1 protein: We

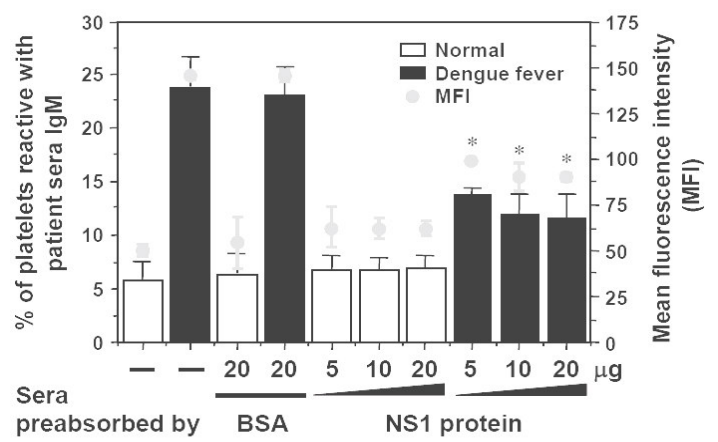

Fig. 1:Competition of DV NS1 protein for the platelet-binding activity of dengue patient sera. Human platelets were incubated with 1:25 dilution of dengue patient or normal control sera pretreated with or without various doses of NS1 or BSA, followed by FITC-conjugated anti-human IgM and analyzed by flow cytometry. Both the percentages and the mean fluorescence intensity (MFI) of platelet-binding activity of antibodies are shown as the mean \pm SD of triplicate cultures. *, $\mathrm{p}<0.05$ as compared with BSA preabsorption group previously found that dengue patients produce anti-platelet and anti-endothelial cell autoantibodies ${ }^{[28,30]}$. We further showed that antibodies against NS1 may, at least in part, account for the cross-reactivity of patient sera with endothelial cells ${ }^{[30]}$. In this study, we investigated whether the platelet-binding activity of dengue patient sera was also due to anti-NS1 antibodies. Patient sera were preabsorbed with various doses of recombinant NS1 or with bovine serum albumin (BSA) as a control. The platelet-binding activity of patient serum IgM was inhibited by pretreatment with NS1 but not BSA, although the inhibitory effect was not complete as compared with the normal control sera (Fig. 1).
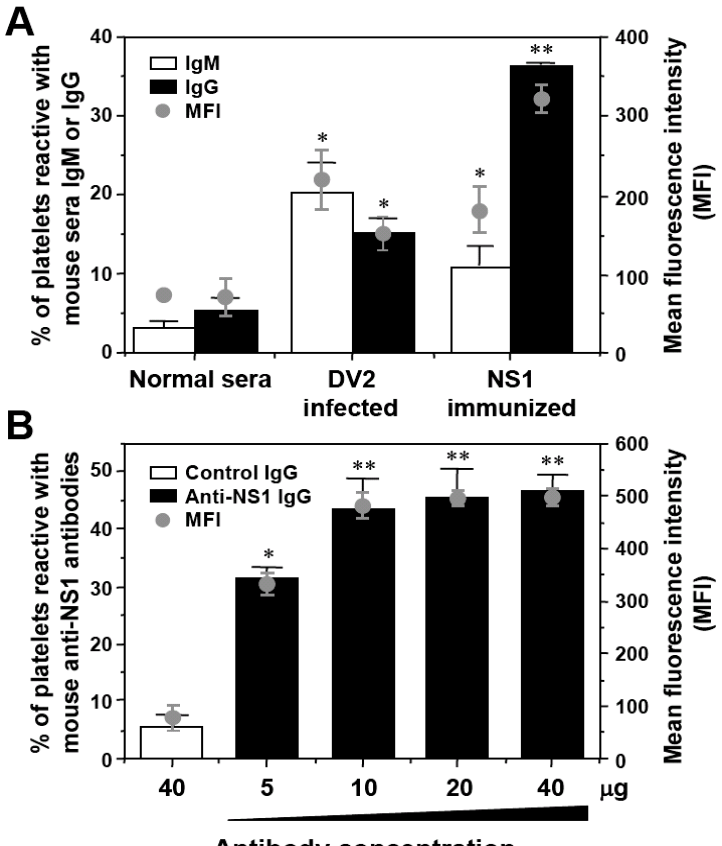

Antibody concentration

Fig. 2:Anti-NS1 antibodies generated in mice cross-react with human platelets. (A) Mice were intravenously injected with DV2 and their sera were collected 8 days later, or intraperitoneally injected with NS1 five times and their sera were collected 4 days after last injection. Human platelets were incubated with 1:25 dilution of DV2-infected, NS1-immunized, or normal control sera, followed by FITC-conjugated anti-mouse $\operatorname{IgM}$ or $\operatorname{IgG}$ and analyzed by flow cytometry. (B) Purified IgG fractions from NS1-immunized or control mouse sera were incubated with human platelets, followed by FITC-conjugated anti-mouse IgG and analyzed by flow cytometry. Both the percentages and the MFI of platelet-binding activity of antibodies are shown as the mean $\pm \mathrm{SD}$ of triplicate cultures. ${ }^{*}, \mathrm{p}<0.05$; $* *, \mathrm{p}<0.01$ as compared with normal sera (A) or control IgG group (B) 
Anti-NS1 antibodies generated in mice cross-react with human platelets: We next tested the platelet-binding activity of DV2-infected or NS1-immunized mouse sera. Both anti-platelet IgM and IgG were present in DV2-infected and NS1-immunized mouse sera (Fig. 2A). NS1-immunized mouse sera were further fractionated to obtain purified IgG. A dose-dependent platelet-binding study of anti-NS1 IgG was shown (Fig. 2B).
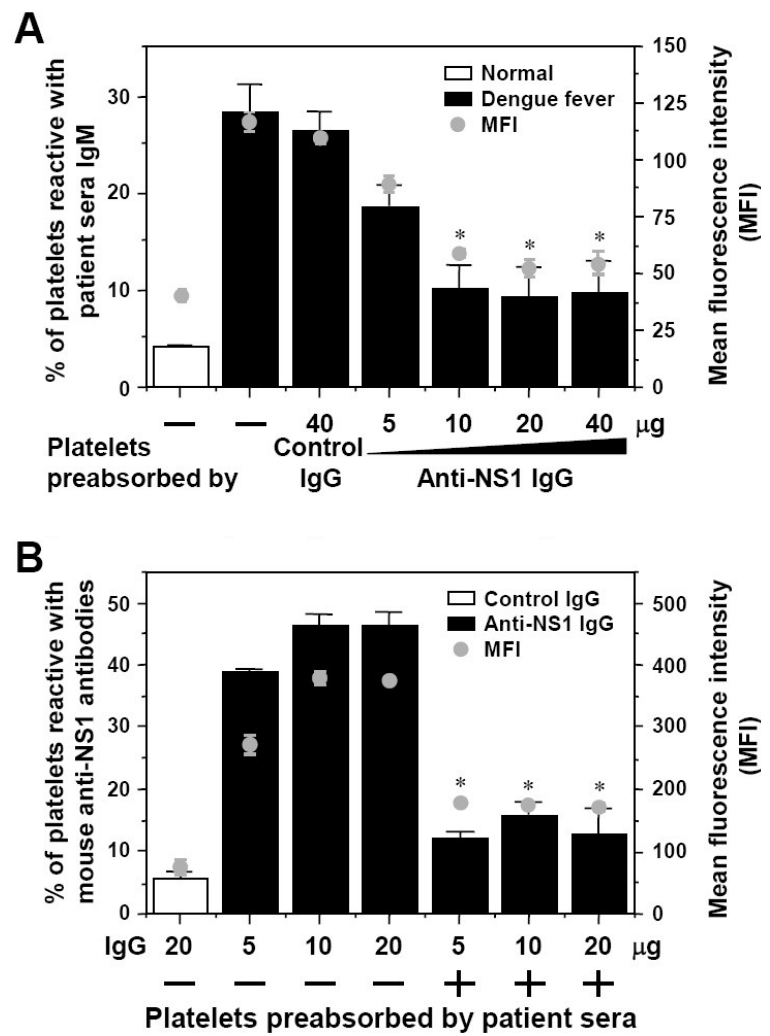

Fig. 3: Platelet-binding activity of dengue patient sera or mouse anti-NS1 antibodies can be inhibited by treatment of platelets by anti-NS1 antibodies or patient sera. Human platelets pretreated with or without various doses of mouse anti-NS1 or control IgG (A) or 1:25 dilution of dengue patient sera (B) were incubated with 1:25 dilution of patient sera (A) or various doses of anti-NS1 IgG (B), followed by FITC-conjugated anti-human IgM or anti-mouse IgG and analyzed by flow cytometry. Both the percentages and the MFI of platelet-binding activity of antibodies are shown as the mean $\pm \mathrm{SD}$ of triplicate cultures. ${ }^{*}, \mathrm{p}<0.05$ as compared with control $\operatorname{IgG}$ (A) or no preabsorption groups (B)
To further investigate the binding activity of anti-NS1 antibodies to platelets, human platelets were preabsorbed with anti-NS1 IgG or patient sera to block the potential cross-reactive molecules on the surface of platelets. As shown in Fig. 3A, the binding activity of dengue patient sera to platelets can be inhibited by pretreatment of platelets with anti-NS1 IgG. Also, pretreatment of platelets with patient sera caused a decrease in platelet-binding activity of anti-NS1 antibodies (Fig. 3B).

Mouse anti-NS1 antibodies inhibit ADP-induced platelet aggregation and cause platelet lysis: Dengue patient sera were shown previously to inhibit platelet aggregation induced by $\mathrm{ADP}^{[28]}$. We thus determined whether the inhibitory effect of platelet aggregation was due to anti-NS1 antibodies. As shown in Fig. 4, anti-NS1 IgG inhibited ADP-induced platelet aggregation as compared with the control IgG.

Complement-mediated platelet cytotoxicity in the presence of patient sera was demonstrated previously ${ }^{[28]}$. Here, we found that anti-NS1 IgG-induced platelet lysis increased dose-dependently with complement (Fig. 5).

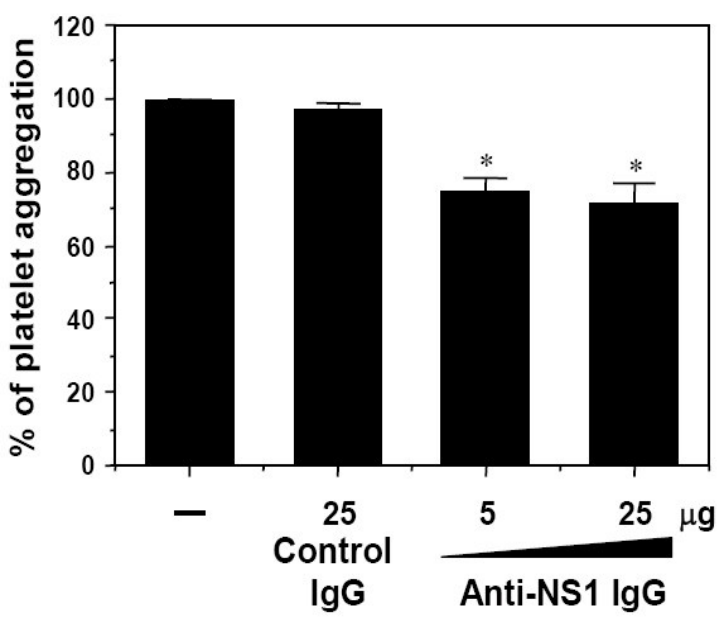

Fig. 4: Anti-NS1 antibodies inhibit ADP-induced platelet aggregation. Various doses of mouse anti-NS1 or control IgG were incubated with platelet-rich plasma and the percentages of platelet aggregation in the presence of ADP were monitored by aggregometry. The normal control without addition of antibodies is normalized to $100 \%$ of platelet aggregation. Data are shown as the mean \pm SD of triplicate cultures. $*, p<0.05$ as compared with control IgG group 


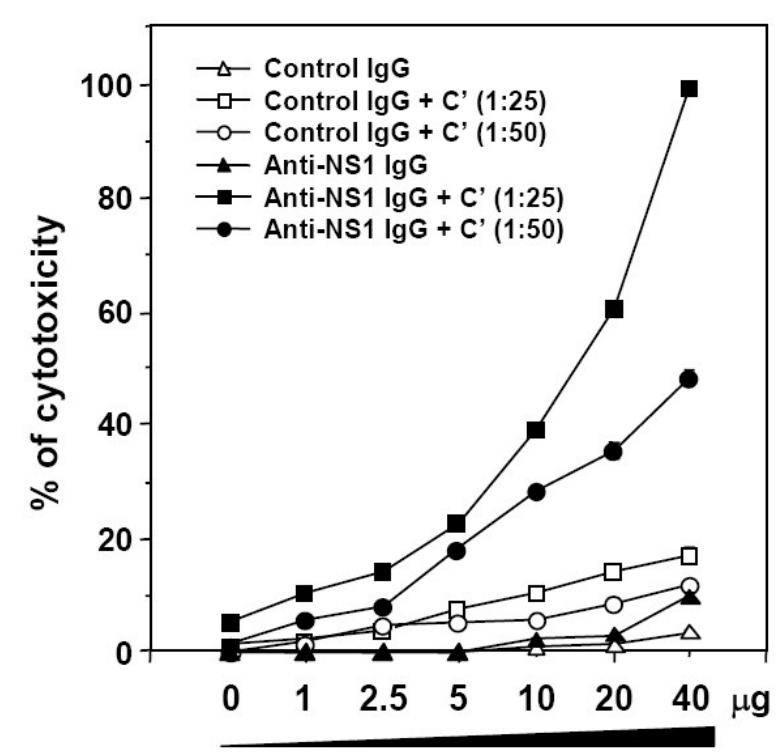

Antibody concentration

Fig. 5: Anti-NS1 antibodies cause platelet lysis in the presence of complement. Various doses of mouse anti-NS1 or control IgG were incubated with or without $1: 25$ or 1:50 dilution of complement before addition to human platelets. After $4 \mathrm{~h}$, the culture supernatant was harvested and the lactate dehydrogenase activity was determined using the Cytotoxicity Detection Kit. Experiments were carried out in duplicate and the mean percentages of cytotoxicity are expressed

Patient sera and mouse anti-NS1 antibodies show increased binding activity and cytotoxicity to activated platelets: The cross-reactivity of patient sera and mouse anti-NS1 antibodies to platelets was examined when platelets were pretreated with or without ADP or thrombin. An increased binding of patient sera (Fig. 6A) or anti-NS1 IgG (Fig. 6B) with activated platelets was observed, suggesting an upregulated expression of platelet surface molecules recognized by anti-NS1. In addition, there was increased cytotoxicity to the activated platelets by patient sera and anti-NS1 IgG (Fig. 6).

\section{DISCUSSION}

In the present study, we showed that anti-NS1 antibodies, at least in part, account for the platelet-binding ability of dengue patient sera. Anti-NS1 antibodies generated in mice further confirmed this issue.
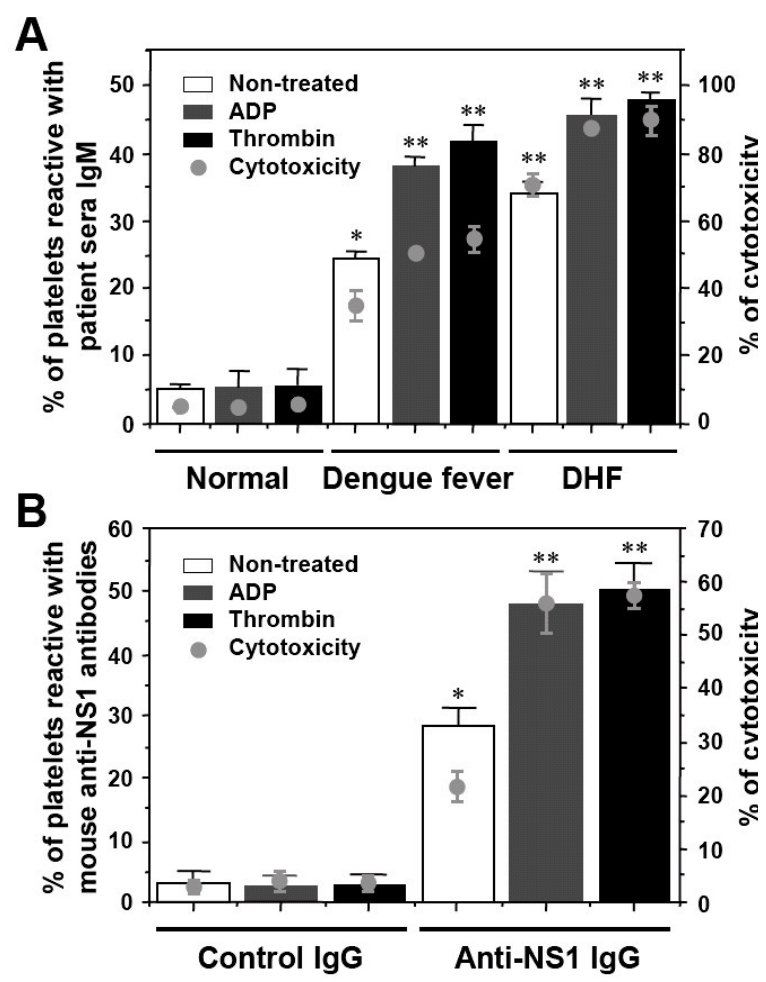

Fig. 6: Platelet-binding activity and cytotoxicity are increased when platelets are activated by ADP or thrombin. Human platelets were activated with or without ADP $(20 \mu \mathrm{M})$ or thrombin $\left(0.1 \mathrm{U} \mathrm{mL}^{-1}\right)$, followed by incubation with 1:25 dilution of dengue fever or DHF patient sera (A) or $5 \mu \mathrm{g}$ of mouse anti-NS1 or control IgG (B) in the presence of 1:25 dilution of complement. The platelet-binding activity was analyzed by flow cytometry and the cytotoxicity was determined using the Cytotoxicity Detection Kit. Both the percentages of platelet-binding activity and complement-mediated cytotoxicity of antibodies are shown as the mean $\pm \mathrm{SD}$ of triplicate cultures. $*, \mathrm{p}<0.05 ; * *, \mathrm{p}<0.01$ as compared with normal sera (A) or control IgG groups (B)

In addition, anti-NS1 antibodies inhibited platelet aggregation and caused platelet lysis in the presence of complement.

Autoimmunity induced by DV infection plays a role in dengue disease ${ }^{[21]}$. We proposed that the presence of anti-platelet autoantibody is related to the pathogenesis of thrombocytopenia. The platelet-binding activity of patient sera was inhibited by preabsorption with DV NS1 protein, although inhibition was not 
complete, as compared with the normal control sera. Therefore, in addition to anti-NS1, other anti-DV antibodies present in patient sera may also be involved in the cross-reactivity with platelets. It should also be noted that the NS1 protein used in these studies was expressed in E. coli and thus would be expected to have conformational differences compared to authentic NS1.

Both DV-infected and NS1-immunized mouse sera showed cross-reactivity with human platelets. Either DV-infection or NS1-immunization induced production of both $\operatorname{IgM}$ and $\mathrm{IgG}$. We used purified $\operatorname{IgG}$ for these studies to avoid the influence of other serum factors. The results of the present study clearly show that such purified IgG shows platelet cross-reactivity. Nevertheless, we previously showed that the isotype of platelet-binding antibodies in dengue patient sera is predominantly $\operatorname{IgM}^{[28]}$. Purified IgM thus also merits future investigation.

Virus-induced thrombocytopenia may be caused by a direct or indirect interaction of the virus with platelets ${ }^{[31,32]}$. A pathological effect of DV infection on the differentiation and growth of human hematopoietic progenitor cells has also been reported ${ }^{[24,25]}$. Host responses to virus infection such as the generation of immune complexes may also cause the platelet destruction. Wang et al. ${ }^{[26]}$ demonstrated that anti-envelope protein antibodies enhanced the platelet-binding ability of DV via non-Fc receptors. In the present study, we showed that anti-NS1 antibodies present in the patient sera or generated in mice cross-react with platelets. Thus, the pathological effects leading to platelet loss are probably due to multiple factors.

Platelet aggregation is suppressed by dengue patient sera ${ }^{[28,33]}$. The induction of platelet lysis in the presence of complement by dengue patient sera has also been demonstrated $^{[28]}$. Further investigation indicated that anti-NS1 antibodies play roles in the inhibition of platelet aggregation and in the complement-mediated platelet lysis. We further showed that the platelet-binding activity and the platelet lysis of dengue patient sera or anti-NS1 antibodies were increased when platelets were preactivated by ADP or thrombin. This can be explained by an increased expression of platelet surface molecules recognized by anti-NS1 antibodies. Various human viruses have been shown to induce autoimmune thrombocytopenia via molecular mimicry to platelet membrane glycoprotein $\mathrm{IIb} / \mathrm{IIIa}^{[34]}$. The identification of the putative platelet surface molecules recognized by anti-NS1 antibodies is a subject of ongoing investigation in our laboratory.

An ideal dengue vaccine should elicit protective immunity but avoid potential pathogenic effects. In order to avoid the damaging effects of ADE, NS1 has been considered a potential target for vaccine development. Protection by antibodies to DV NS1 is thought to involve the recognition of NS1 expressing on the infected cell surface, followed by complement-mediated cell lysis. These positive effects must however be tempered with observations that antibodies against NS1 protein cross-react with platelets, endothelial cells, or blood clotting factors that may result in vascular dysfunction leading to dengue-associated hemorrhage ${ }^{[19,28,30]}$. Using genetic modifications to truncate or mutate the cross-reactive epitopes may provide valuable information for development of safe and effective dengue vaccine. The epitopes shared between DV NS1 protein and platelet surface autoantigens need to be analyzed.

\section{ACKNOWLEDGMENT}

This study was supported by grant NSC95-3112-B006-002 from the National Research Program of Genomic Medicine, National Science Council, Taiwan.

\section{REFERENCES}

1. Henchal, E.A. and J.R. Putnak, 1990. The dengue viruses. Clin. Microbiol. Rev., 3: 376-396.

2. Gubler, D.J., 1998. Dengue and dengue hemorrhagic fever. Clin. Microbiol. Rev., 11: 480-496.

3. Halstead, S.B., 1988. Pathogenesis of dengue: challenges to molecular biology. Science, 239: 476-481.

4. Halstead, S.B., 2002. Dengue. Curr. Opin. Infect. Dis., 15: 471-476.

5. Morens, D.M., 1994. Antibody-dependent enhancement of infection and the pathogenesis of viral disease. Clin. Infect. Dis., 19: 500-512.

6. Halstead, S.B., 2003. Neutralization and antibody-dependent enhancement of dengue viruses. Adv. Virus Res., 60: 421-467.

7. Mady, B.J., D.V. Erbe, I. Kurane, M.W. Fanger and F.A. Ennis, 1991. Antibody-dependent enhancement of dengue virus infection mediated by bispecific antibodies against cell surface molecules other than Fc $\gamma$ receptors. J. Immunol., 147: 3139-3144.

8. Huang, K.J., Y.C. Yang, Y.S. Lin, J.H. Huang, H.S. Liu, T.M. Yeh, S.H. Chen, C.C. Liu and H.Y. Lei, 2006. The dual-specific binding of dengue virus and target cells for the antibody-dependent enhancement of dengue virus infection. J. Immunol., 176: 2825-2832.

9. Rothman, A.L. and F.A. Ennis, 1999. Immunopathogenesis of dengue hemorrhagic fever. Virology, 257: 1-6. 
10. Lei, H.Y., T.M. Yeh, H.S. Liu, Y.S. Lin, S.H. Chen and C.C. Liu, 2001. Immunopathogenesis of dengue virus infection. J. Biomed. Sci., 8: 377-388.

11. Green, S. and A. Rothman, 2006. Immunopathological mechanisms in dengue and dengue hemorrhagic fever. Curr. Opin. Infect. Dis., 19: 429-436.

12. Clyde K, J.L. Kyle and E. Harris, 2006. Recent advances in deciphering viral and host determinants of dengue virus replication and pathogenesis. J. Virol., 80: 11418-11431.

13. Rothman, A.L., 2004. Dengue: defining protective versus pathologic immunity. J. Clin. Invest., 113: 946-951.

14. King, C.A., J.S. Marshall, H. Alshurafa and R. Anderson, 2000. Release of vasoactive cytokines by antibody-enhanced dengue virus infection of a human mast cell/basophil line. J. Virol., 74: 7146-7150.

15. Malasit, P., 1987. Complement and dengue haemorrhagic fever/shock syndrome. Am. J. Trop. Med. Hyg., 18: 316-320.

16. Avirutnan, P., P. Malasit, B. Seliger, S. Bhakdi and M. Husmann, 1998. Dengue virus infection of human endothelial cells leads to chemokine production, complement activation and apoptosis. J. Immunol., 161: 6338-6346.

17. Green. S., D.W. Vaughn, S. Kalayanarooj, S. Nimmannitya, S. Suntayakorn, A. Nisalak, R. Lew, B.L. Innis, I. Kurane, A.L. Rothman and F.A. Ennis, 1999. Early immune activation in acute dengue illness is related to development of plasma leakage and disease severity. J. Infect. Dis., 179: 755-762.

18. Mongkolsapaya, J., W. Dejnirattisai, X.N. Xu, S. Vasanawathana, N. Tangthawornchaikul, A. Chairunsri, S. Sawasdivorn, T. Duangchinda, T. Dong, S. Rowland-Jones, P. Yenchitsomanus, A. McMichael, P. Malasit and G. Screaton, 2003. Original antigenic sin and apoptosis in the pathogenesis of dengue hemorrhagic fever. Nat. Med., 9: 921-927.

19. Falconar, A.K.I., 1997. The dengue virus nonstructural-1 protein (NS1) generates antibodies to common epitopes on human blood clotting, integrin/adhesin proteins and binds to human endothelial cells: potential implications in haemorrhagic fever pathogenesis. Arch. Virol., 142: 897-916.

20. Chaturvedi, U.C., E.A. Elbishbishi, R. Agarwal and A.S. Mustafa, 2001. Cytotoxic factor-autoantibodies: possible role in the pathogenesis of dengue haemorrhagic fever. FEMS Immunol. Med. Microbiol., 30: 181-186.

21. Lin, C.F., S.W. Wan, H.J. Cheng, H.Y. Lei and Y.S. Lin, 2006. Autoimmune pathogenesis in dengue virus infection. Viral Immunol., 19: 127-132.

22. Rigau-Perez, J.G., G.G. Clark, D.J. Gubler, P. Reiter, E.J. Sanders and A.V. Vorndam, 1998. Dengue and dengue haemorrhagic fever. Lancet, 352: 971-977.
23. Hathirat, P., P. Isarangkura, T. Srichaikul, V. Suvatte and C. Mitrakul, 1993. Abnormal hemostasis in dengue hemorrhagic fever. Southeast Asian J. Trop. Med. Public Health, 24: 80-85.

24. Nakao, S., C.J. Lai and N.S. Young, 1989. Dengue virus, a flavivirus, propagates in human bone marrow progenitors and hematopoietic cell lines. Blood, 74: 1235-1240.

25. Murgue, B., O. Cassar, M. Guigon and E. Chungue, 1997. Dengue virus inhibits human hematopoietic progenitor growth in vitro. J. Infect. Dis., 175: 1497-1501.

26 Wang, S., R. He, J. Patarapotikul, B.L. Innis and R. Anderson, 1995. Antibody-enhanced binding of dengue-2 virus to human platelets. Virology, 213: 254-257.

27. Butthep, P., A. Bunyaratvej and N. Bhamarapravati, 1993. Dengue virus and endothelial cell: a related phenomenon to thrombocytopenia and granulocytopenia in dengue hemorrhagic fever. Am. J. Trop. Med. Hyg., 24: 246-249.

28. Lin, C.F., H.Y. Lei, C.C. Liu, H.S. Liu, T.M. Yeh, S.T. Wang, T.I. Yang, F.C. Sheu, C.F. Kuo and Y.S. Lin, 2001. Generation of IgM anti-platelet autoantibody in dengue patients. J. Med. Virol., 63: 143-149.

29. Huang, K.J., S.Y. Li, S.C. Chen, H.S. Liu, Y.S. Lin, T.M. Yeh, C.C. Liu and H.Y. Lei, 2000. Manifestation of thrombocytopenia in dengue-2-virus-infected mice. J. Gen. Virol., 81: 2177-2182.

30. Lin, C.F., H.Y. Lei, A.L. Shiau, C.C. Liu, H.S. Liu, T.M. Yeh, S.H. Chen and Y.S. Lin, 2003. Antibodies from dengue patient sera cross-react with endothelial cells and induce damage. J. Med. Virol., 69: 82-90.

31. van Gorp, E.C.M., C. Suharti, H. ten Cate, W.M.V. Dolmans, J.W.M. van der Meer, J.W. ten Cate and D.P.M. Brandjes, 1999. Review: infectious diseases and coagulation disorders. J. Infect. Dis., 180: 176-186.

32. Srichaikul, T., S. Nimmannitya, T. Sripaisarn, M. Kamolsilpa and C. Pulgate, 1989. Platelet function during the acute phase of dengue hemorrhagic fever. Southeast Asian J. Trop. Med. Public Health, 20: 19-25.

33. Kaplan, C., F. Morinet and J. Cartron, 1992. Virus-induced autoimmune thrombocytopenia and neutropenia. Semin. Hematol., 29: 34-44.

34. Boughton, B.J., A.W. Simpson, C. Bolt, A. Buchan and P. McLeish, 1995. Platelet membrane glycoprotein IIb/IIIa has sequence homologies with human virus proteins and synthetic viral peptides inhibit anti-GPIIb/IIIa antibodies in autoimmune thrombocytopenic purpura. Platelets, 6: 75-82. 